\title{
Lymphocyte-to-monocyte ratio before chemoradiotherapy represents a prognostic predictor for locally advanced rectal cancer
}

This article was published in the following Dove Press journal:

OncoTargets and Therapy

Number of times this article has been viewed

\author{
Yu-Xiang Dengl,* \\ Jun-Zhong Lin ${ }^{1, *}$ \\ Jian-Hong Peng ${ }^{1, *}$ \\ Yu-Jie Zhao' \\ Qiao-Qi Sui' \\ Xiao-Jun Wu' \\ Zhen-Hai Lu' \\ Yuan-Hong Gao \\ Zhi-Fang Zeng ${ }^{2}$ \\ Zhi-Zhong Pan' \\ 'Department of Colorectal Surgery, \\ ${ }^{2}$ Department of Radiation Oncology, \\ Sun Yat-sen University Cancer Center, \\ State Key Laboratory of Oncology in \\ South China, Collaborative Innovation \\ Center for Cancer Medicine \\ Guangzhou, Guangzhou, People's \\ Republic of China \\ *These authors contributed equally \\ to this work
}

\begin{abstract}
Research indicates that cancer-triggered inflammation plays a pivotal role in carcinogenesis. Here, we aimed to evaluate the correlation of lymphocyte-to-monocyte ratio (LMR) before neoadjuvant chemoradiotherapy (CRT) with clinical outcomes in patients with locally advanced rectal cancer (LARC). We retrospectively enrolled 317 consecutive patients with LARC between 2004 and 2013. The optimal cutoff values of LMR were determined using receiver operating curve analysis. Overall survival (OS) and disease-free survival related to the LMR were analyzed using the log-rank test and multivariate Cox regression methods. We found that a low LMR $(\leq 4.91)$ was prominently correlated with worse prognostic features and a shorter 3-year survival rate of LARC. Moreover, multivariate Cox analysis revealed that elevated LMR was an independent factor for better OS (hazard ratio $0.538,95 \%$ confidence interval $0.292-0.991, P=0.047$ ). In addition, univariate logistic regression analysis showed that the LMR was not associated with tumor pathologic regression. In conclusion, LMR is identified as a valuable prognostic marker for predicting the OS of LARC patients receiving CRT.
\end{abstract}

Keywords: rectal cancer, lymphocyte-monocyte ratio, prognosis, systemic inflammation

\section{Introduction}

Colorectal cancer (CRC) is the third most commonly diagnosed cancer and the fourth leading cause of cancer-related mortality worldwide. ${ }^{1}$ Rectal cancer accounts for approximately $30 \%$ of CRC and is associated with worse clinical outcomes. ${ }^{2,3}$ Current treatment guidelines for locally advanced rectal cancer (LARC) include preoperative chemoradiotherapy (CRT), total mesorectal excision (TME) and postoperative adjuvant chemotherapy. ${ }^{4}$ CRT combined with TME has been shown to significantly reduce the rate of local disease recurrence and improve the rectal cancer sphincter preservation rate. ${ }^{5}$ However, not all patients benefit from CRT; ${ }^{6}$ thus, identifying biomarkers that can predict the efficacy of CRT is essential for decision making in the management of patients with CRC. Research has shown that reduced E-cadherin, nucleic beta-catenin and reduced expression of mir200c were all significantly associated with nonresponse to neoadjuvant therapy, ${ }^{7}$ but the inspection method is expensive. Previous studies have suggested that inflammation plays a critical role in the carcinogenesis and progression of cancer. ${ }^{8,9}$ In addition, it has recently been reported that the lymphocyte-to-monocyte ratio (LMR) is an independent predictor of overall survival (OS) in patients with $\mathrm{CRC}$ undergoing curative resection, ${ }^{10}$ and studies have reported that the preoperative LMR is a simple and useful prognostic indicator in patients with stage IV CRC who have undergone curative resection. ${ }^{11}$ However, evidence of
Correspondence: Zhi-Zhong Pan

Department of Colorectal Surgery, Sun Yat-sen University Cancer Center, 65I Dongfeng Road East, 510060 Guangzhou, People's Republic of China

Tel +86 2087343124

Fax +86 2087343637

Email panzhzh@sysucc.org.cn (c)
hereby accept the Terms. Non-commercial uses of the work are permitted without any further permission from Dove Medical Press Limited, provided the work is properly attributed. For permission for commercial use of this work, please see paragraphs 4.2 and 5 of our Terms (https://www.dovepress.com/terms.php). 
preoperative LMR as a prognostic predictor in patients with LARC receiving CRT remains limited. Accordingly, this study investigated the association between LMR before CRT and clinical outcomes in patients with LARC.

\section{Materials and methods}

\section{Patients}

The present study retrospectively enrolled consecutive patients undergoing primary tumor resection and preoperative CRT with curative intent from April 2004 to June 2013 at Sun Yat-sen University Cancer Center, People's Republic of China. The enrolled patients met the following inclusion criteria: 1) histologically confirmed adenocarcinoma; 2) T3-4 or N+ disease initially; 3) preoperative chemotherapy with XELOX regimen (capecitabine plus oxaliplatin); and 4) completed radical resection. Patients were excluded from the analysis if they had metastatic disease before or during preoperative treatment, had other active malignancies, died within 1 month postoperatively, or had missing preoperative data on the number of blood cells. Patient demographics, primary tumor characteristics, preoperative treatment, and follow-up results were reviewed in detail from the medical records and the follow-up system. The present study was undertaken according to the ethical standards of the World Medical Association Declaration of Helsinki. Institutional Review Board approval was obtained from independent ethics committees at Sun Yat-sen University Cancer Center, with a waiver of informed consent as this research was retrospective and did not involve accessing any identifying patient data. The raw data in this paper has been successfully uploaded and locked onto Research Data Deposit with a RDD number of RDDA2017000378.

\section{CRT and surgery}

Patients were evaluated using a combination of colonoscopy, computed tomography (CT), ultrasonography, and magnetic resonance imaging (MRI) before CRT. Tumor stage was classified according to the seventh edition of the American Joint Committee on Cancer tumor node metastasis classification system. One to four cycles of XELOX regimen included oxaliplatin at $130 \mathrm{mg} / \mathrm{m}^{2}$ on Day 1 and capecitabine at $1,000 \mathrm{mg} / \mathrm{m}^{2}$ twice daily on Days $1-14$ with an interval of 7 days. Radiotherapy consisted of 46.0-50.0 Gy delivered in 23-25 fractions of 2 Gy five times per week. The target field included the tumor bed, the surrounding intestines, and the presacral and iliac lymphatic drainage areas. Radical surgical resection was planned for 6-8 weeks after completing CRT. The primary tumor surgical types included Dixon, Miles,
TME, and other surgical procedures. Each surgical resection specimen was reviewed by an experienced pathologist. Pathologic response was classified in five categories as proposed by Mandard et al: ${ }^{12}$ tumor regression grade (TRG) 1 , no residual cancer cells; TRG 2, rare residual cancer cells; TRG 3, fibrosis outgrowing residual cancer; TRG 4, residual cancer outgrowing fibrosis; and TRG 5, absence of regressive change.

\section{Inflammatory index calculation}

The systemic inflammatory data (lymphocyte and monocyte counts), carcinoembryonic antigen (CEA) and cancer antigen 199 (CA199) were collected from routine blood tests that were taken before neoadjuvant therapy. Neutrophil and lymphocyte counts were determined by routine blood test using Sysmex XE-5000TM Automated Hematology System (Sysmex, Shanghai, People's Republic of China). The LMR was calculated by dividing the absolute number of circulating lymphocytes by the absolute number of monocytes. CEA and CA199 were detected using electrochemiluminescence immunoassay system (Elecsys 1601; Roche, Basel, Switzerland), and the cutoff values for CEA and CA199 were $5 \mathrm{ng} / \mathrm{mL}$ and $35 \mathrm{U} / \mathrm{mL}$, respectively, according to the manufacturer's instructions. The values lower than the cutoff values were considered negative.

\section{Follow-up}

The follow-up protocol included evaluations every 3 months for the first 2 years after the completion of surgery, every 6 months for the third to fifth years and then once every year thereafter. Evaluations at each visit included complete blood count, CEA and CA199 measurements, and physical examination. Chest radiography, abdominal and pelvic CT, pelvic endoscopic ultrasonography or MRI were conducted every 12 months during follow-up. Colonoscopy was performed annually. OS was defined as the time from the date of surgery to the date of death or last follow-up, and diseasefree survival (DFS) was defined as the time from the date of surgery to the date of disease recurrence or last follow-up. All follow-up statistics were reviewed by December 31, 2016.

\section{Statistical analysis}

The statistical analysis was performed using the IBM SPSS Statistics 20.0 software (SPSS, Chicago, IL, USA) and GraphPad Prism 7 software (GraphPad Software, Inc, San Diego, CA, USA). Receiver operating curve (ROC) analysis was performed to evaluate the sensitivity and specificity of the LMR for predicting 3-year OS. The Youden index was 
estimated to select the optimal cutoff value of the LMR. We compared continuous variables that were normally distributed using Student's $t$-test and categorical variables using the chi square test or Fisher's test. The Kaplan-Meier method was used to estimate the survival rates for different groups, and the differences in the survival curves were compared with the log-rank test. The Cox proportional hazards model and the logistic regression model were used for univariate and multivariate survival analyses. $P<0.05$ was considered statistically significant.

\section{Results}

\section{Patient characteristics and determination of the cutoff point}

The optimal cutoff value of the LMR was 4.91, corresponding to maximum sensitivity and specificity (0.464 and 0.731 , respectively) of the LMR for predicting 3-year OS in ROC analysis, and the Youden index was 0.182 . The area under the curve was 0.584 for 3 -year OS (95\% confidence interval [CI], 0.513-0.654, $P=0.036)$. Patients were then assigned either to the LMR-high (LMR >4.91) group or the LMR-low (LMR $\leq 4.91)$ group (Figure 1).

Among the 317 LARC patients, 64.7\% (205/317) were males and $35.3 \%$ (112/317) were females, the median age was 55 years (range 15-78 years) and the average completed cycles of XELOX was 2.54 (range 1-8). In addition, among

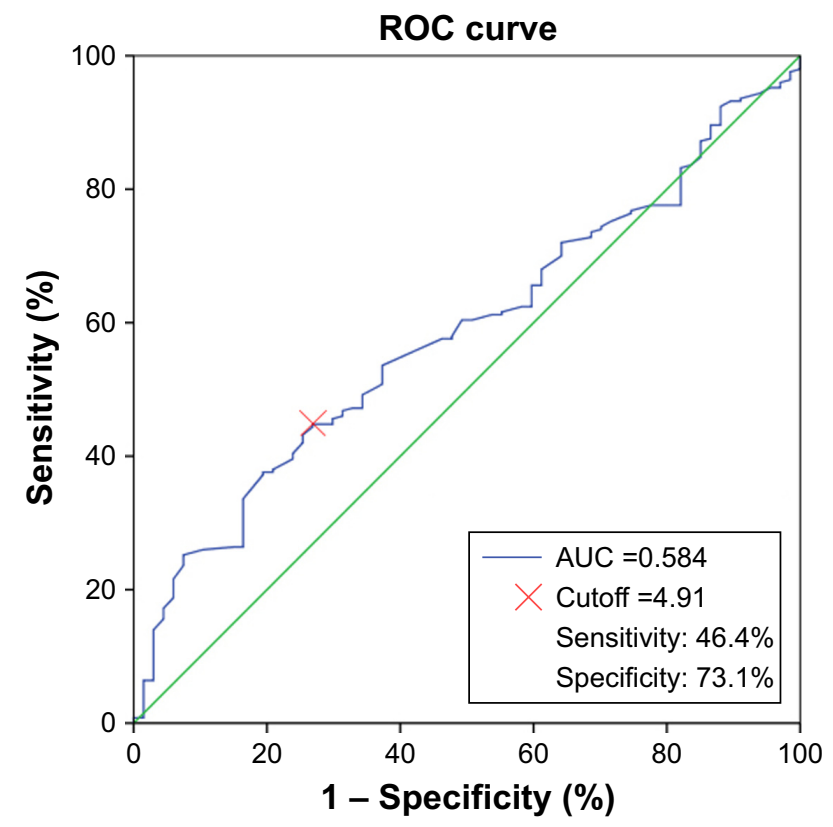

Figure I ROC for determination of the cutoff value for the LMR in patients with locally advanced rectal cancer with preoperative chemoradiotherapy.

Abbreviations: ROC, receiver operating curve; LMR, lymphocyte-to-monocyte ratio; AUC, area under the curve. the patients $34.3 \%(85 / 317)$ had a complete pathologic response, $25 \%(62 / 317)$ were diagnosed with stage 1 disease, $40.7 \%(101 / 317)$ were diagnosed with stage 2 disease and $21.8 \%$ (69/317) were diagnosed with stage 3 disease. The median interval days from radiotherapy to surgery was 49 days (range $7-168$ days).

The associations of baseline characteristics with 3-year OS in LARC patients are listed in Table 1. As shown in Table 1, the high-LMR group had more female patients $(P=0.022)$ and more cycles of XELOX treatment before surgery $(P=0.002)$. Additionally, a higher proportion of the high-LMR group received intensity-modulated radiation therapy $(P=0.002)$, and 50 Gy of radiotherapy dose $(P=0.001)$.

\section{Association between patient characteristics and tumor pathologic regression}

The association between patient characteristics and tumor pathologic regression after CRT is shown in Table 2. The univariate logistic regression analysis showed that the LMR was not associated with tumor pathologic regression. The multivariate analysis showed that well-differentiated histology (odds ratio, $7.453 ; 95 \%$ CI, 1.911-17.662; $P=0.002$ ) was associated with better pathologic response.

\section{Analysis of the prognostic impact of the LMR on OS and DFS}

To identify the association of the LMR before CRT with the clinical outcomes in patients with LARC, we evaluated the LMR and clinicopathologic factors in univariate and multivariate analyses. With a median follow-up time of 51 months (range 1-109 months), six (1.9\%) patients were identified as having local recurrence, $68(21.5 \%)$ patients were identified as having distant metastasis (liver metastases, 11; lung metastasis, 35; pelvic cavity metastasis, eight; bone metastases, eight; and other metastases, 11), 57 (21.1\%) patients had cancer-related deaths and 10 (3.2\%) were other causes of death. Kaplan-Meier analysis indicated that the 3-year OS rate in the high-LMR group was significantly higher than that in the low-LMR group ( $87.9 \%$ vs $74.3 \%, P=0.019$; Figure 2A). By contrast, the 3-year DFS rates of the low- and high-LMR groups were $73.3 \%$ and $83.2 \%$, respectively, and this rate was not significantly different between the subgroups ( $P=0.096$; Figure 2B).

The univariate analysis revealed that the higher LMR was associated with better 3-year OS rates (hazard ratio [HR], 0.493; 95\% CI, 0.269-0.904; $P=0.022$ ) (Table 3). In addition, 
Table I Clinical characteristics of the patients stratified by LMR

\begin{tabular}{|c|c|c|c|c|}
\hline \multirow[t]{2}{*}{ Characteristics } & \multirow{2}{*}{$\begin{array}{l}\text { Total } \\
n=317 \text { (\%) }\end{array}$} & \multicolumn{3}{|l|}{ LMR } \\
\hline & & $\leq 4.91$ & $>4.91$ & $P$-value \\
\hline \multicolumn{4}{|l|}{ Age (years) } & 0.538 \\
\hline$\leq 60$ & $212(66.9)$ & 138 & 74 & \\
\hline$>60$ & $105(33.1)$ & 72 & 33 & \\
\hline \multicolumn{4}{|l|}{ Sex } & 0.022 \\
\hline Male & $205(64.7)$ & 145 & 60 & \\
\hline Female & $112(35.3)$ & 65 & 47 & \\
\hline \multicolumn{4}{|c|}{ Cycles of neoadjuvant XELOX regimen } & $<0.002$ \\
\hline I & $13(4.1)$ & 10 & 3 & \\
\hline 2 & $162(51.1)$ & 117 & 45 & \\
\hline 3 & $63(19.9)$ & 42 & 21 & \\
\hline 4 & 79 (24.9) & 41 & 38 & \\
\hline \multicolumn{4}{|l|}{ Radiotherapy model } & $<0.002$ \\
\hline IMRT & $154(48.6)$ & 87 & 67 & \\
\hline 3D-CRT & $146(46.1)$ & 110 & 36 & \\
\hline Conventional RT & $17(5.4)$ & 13 & 4 & \\
\hline \multicolumn{4}{|l|}{ Radiation dose (Gy) } & 0.001 \\
\hline 46 & $17 \mid(53.9)$ & 127 & 44 & \\
\hline 50 & $146(46.1)$ & 83 & 63 & \\
\hline \multicolumn{4}{|c|}{ Interval from radiotherapy to surgery (days) } & 0.174 \\
\hline$\leq 49$ & $210(66.2)$ & 117 & 93 & \\
\hline$>49$ & $107(33.8)$ & 51 & 56 & \\
\hline \multicolumn{4}{|l|}{ Tumor size $(\mathrm{cm})$} & 0.133 \\
\hline$\leq 3$ & $226(71.3)$ & 144 & 82 & \\
\hline$>3$ & $91(28.7)$ & 66 & 25 & \\
\hline \multicolumn{4}{|l|}{$\mathrm{DAV}(\mathrm{cm})$} & 0.176 \\
\hline$\leq 5$ & $165(52.1)$ & 115 & 50 & \\
\hline$>5$ & $152(47.9)$ & 95 & 57 & \\
\hline \multicolumn{4}{|l|}{ Postoperative TNM stage } & $<0.191$ \\
\hline $\mathrm{pCR}$ & $85(26.8)$ & 53 & 32 & \\
\hline i & $62(19.6)$ & 40 & 22 & \\
\hline IIA & $59(18.6)$ & 35 & 24 & \\
\hline IIB & $42(13.2)$ & 35 & 7 & \\
\hline IIIA & $16(5.0)$ & 7 & 9 & \\
\hline IIIB & $48(15.1)$ & 36 & 12 & \\
\hline IIIC & $5(2.0)$ & 3 & 2 & \\
\hline \multicolumn{4}{|l|}{ TRG } & 0.097 \\
\hline $\mathrm{I}-2$ & $160(50.5)$ & 99 & 61 & \\
\hline $3-5$ & $157(49.5)$ & III & 46 & \\
\hline \multicolumn{4}{|l|}{ Surgical type } & $<0.064$ \\
\hline Anterior resection & $203(64.0)$ & 125 & 78 & \\
\hline Abdominal perineal resection & $106(33.4)$ & 79 & 27 & \\
\hline Others & $8(2.5)$ & 6 & 2 & \\
\hline \multicolumn{4}{|l|}{ Tumor differentiation } & $0.84 I$ \\
\hline Well differentiated & $34(10.7)$ & 22 & 12 & \\
\hline Moderately or poorly & $283(89.3)$ & 188 & 95 & \\
\hline \multicolumn{5}{|l|}{ differentiated } \\
\hline Pre-CRT CEA (ng/mL) & & & & 0.528 \\
\hline$\leq 5$ & $161(50.8)$ & 104 & 57 & \\
\hline$>5$ & $156(49.2)$ & 106 & 50 & \\
\hline Pre-CRT CAI99 (U/mL) & & & & 0.120 \\
\hline$\leq 35$ & $264(83.3)$ & 170 & 94 & \\
\hline$>35$ & $53(16.7)$ & 40 & 13 & \\
\hline
\end{tabular}

Note: $P$-value, the correlation between clinical-pathological parameters and LMR was assessed by the chi-square test or Fisher's exact test.

Abbreviations: LMR, lymphocyte-to-monocyte ratio; XELOX, oxaliplatin $130 \mathrm{mg} / \mathrm{m}^{2}$ administered intravenously on Day I and capecitabine administered orally I,000 mg/m² twice daily on Days I-14 for a 3-week cycle; IMRT, intensitymodulated radiation therapy; 3D-CRT, 3-dimensional conformal radiation therapy; $\mathrm{RT}$, radiotherapy; DAV, distance of inferior tumor margin from the anal verge; TNM, tumor node metastasis; PCR, pathologic complete response; TRG, tumor regression grade; CRT, chemoradiotherapy; CEA, carcinoembryonic antigen; CA199, cancer antigen 199. tumor size less than $3 \mathrm{~cm}$, I/II stage, $1-2$ of tumor regression grading and preoperative CA199 less than $35 \mathrm{U} / \mathrm{mL}$ were associated with better 3 -year OS rates of LARC. On the other hand, tumor size less than $3 \mathrm{~cm}$, I/II stage, $1-2$ of tumor regression grading and preoperative CA199 less than $35 \mathrm{U} / \mathrm{mL}$ were associated with better 3-year DFS rates of LARC.

The multivariate analysis showed that I/II stage (HR, 3.004; 95\% CI, 1.862-4.959; $P<0.001), \mathrm{CA} 199 \leq 35 \mathrm{U} / \mathrm{mL}(\mathrm{HR}$, 1.906; 95\% CI, 1.116-3.254; $P=0.018$ ) and LMR $>4.91$ (HR, $0.538 ; 95 \% \mathrm{CI}, 0.292-0.991 ; P=0.047)$ were significant prognostic factors for 3-year OS rate. Similarly, tumor $\leq 3 \mathrm{~cm}$ (HR, $1.927 ; 95 \% \mathrm{CI}, 1.214-3.059 ; P=0.005)$ and $\mathrm{CA} 199 \leq 35 \mathrm{U} / \mathrm{mL}$ (HR, 3.182; 95\% CI, 1.996-5.074; $P<0.001)$ were also defined as independent factors for 3-year DFS rate.

\section{Discussion}

Nearly 150 years ago, Rudolf Virchow speculated that cancer is similar to the chronic inflammatory process of wound healing, as he noticed the presence of a large amount of leukocyte infiltration in tumor tissues. ${ }^{8}$ Epidemiologic and clinical studies support his notion and show that approximately $25 \%$ of all human cancers in adults result from chronic inflammation. ${ }^{13}$ Research has shown that inflammation plays an important role in the onset, development and therapeutic response of many tumors. It contributes to tumor proliferation, angiogenesis and metastasis and it defeats the adaptive immune responses..$^{9,10,14}$

Systemic inflammation is achieved by the increased movement of plasma and leukocytes (includes lymphocytes and monocytes) from the blood into the inflamed tissue. Research has shown that cytotoxic $\mathrm{T}$ cells use two basic mechanisms to destroy their target cells: one is elicited by granule exocytosis (perforin [PRF1] and granule-associated enzymes [granzymes, GZM]) and the other occurs via the death ligand/death receptor system and is ultimately responsible for killing the cancer cells and eradicating the tumor. ${ }^{15}$ Lymphocytes are key factors in immunosurveillance, and the occurrence of an immunologic antitumor reaction depends on lymphocytic infiltration into the tumor microenvironment. ${ }^{16-18}$ However, tumor-induced systemic inflammation will depress cellular immunity significantly, resulting in a significant decrease of $\mathrm{CD}^{+} \mathrm{T}$ lymphocytes and an increase of $\mathrm{CD} 8^{+}$ suppressor T lymphocytes. ${ }^{19}$ Researchers have found that the lymphocyte-mediated immune reaction may play a positive role in achieving the complete eradication of tumor cells. ${ }^{20}$ Accordingly, for patients with rectal cancer who underwent CRT, maintaining the number of lymphocytes is beneficial to the prognosis of the patients. ${ }^{21}$ On the other hand, Augier 
Table 2 The univariate and multivariate analyses of the factors influencing tumor pathologic regression response in locally advanced rectal cancer after preoperative chemoradiotherapy

\begin{tabular}{|c|c|c|c|c|}
\hline & \multicolumn{2}{|l|}{ Univariate analysis } & \multicolumn{2}{|l|}{ Multivariate analysis } \\
\hline & OR $(95 \% \mathrm{Cl})$ & $P$-value & OR $(95 \% \mathrm{Cl})$ & $P$-value \\
\hline \multicolumn{5}{|l|}{ Age (years) } \\
\hline$\leq 60$ & I (reference) & & - & \\
\hline$>60$ & $0.94 \mid(0.556-|.59|)$ & 0.820 & - & \\
\hline \multicolumn{5}{|l|}{ Sex } \\
\hline Male & I (reference) & & - & \\
\hline Female & $0.934(0.557-1.568)$ & 0.797 & - & \\
\hline \multicolumn{5}{|l|}{ Cycles of preoperative XELOX regimen } \\
\hline $\mathrm{I}-2$ & I (reference) & & - & \\
\hline $3-4$ & $0.883(0.536-1.453)$ & 0.624 & - & \\
\hline \multicolumn{5}{|l|}{ Radiotherapy model } \\
\hline IMRT & I (reference) & & - & \\
\hline 3D-CRT & $1.504(0.896-2.524)$ & 0.122 & - & \\
\hline Conventional RT & $1.054(0.352-3.16 \mathrm{I})$ & 0.925 & - & \\
\hline \multicolumn{5}{|l|}{ Radiation dose (Gy) } \\
\hline 46 & I (reference) & & - & \\
\hline 50 & $0.685(0.416-1.129)$ & 0.138 & - & \\
\hline \multicolumn{5}{|c|}{ Interval from radiotherapy to surgery (days) } \\
\hline$\leq 49$ & I (reference) & & - & \\
\hline$>49$ & $0.677(0.4 \mid I-I .115)$ & 0.125 & - & \\
\hline \multicolumn{5}{|l|}{ Tumor size $(\mathrm{cm})$} \\
\hline$\leq 3$ & I (reference) & & - & \\
\hline$>3$ & $2.060(1.120-3.789)$ & 0.020 & - & \\
\hline \multicolumn{5}{|l|}{$\mathrm{DAV}(\mathrm{cm})$} \\
\hline$\leq 5$ & I (reference) & & - & \\
\hline$>5$ & $1.050(0.638-1.727)$ & 0.848 & - & \\
\hline \multicolumn{5}{|l|}{ Clinical TNM stage } \\
\hline $\mathrm{l} / \mathrm{II}$ & I (reference) & & - & \\
\hline III & $1.420(0.832-2.423)$ & 0.199 & - & \\
\hline \multicolumn{5}{|l|}{ Surgical type } \\
\hline Anterior resection & I (reference) & 0.289 & - & \\
\hline Abdominal perineal resection & $\mathrm{I} .434(0.83 \mathrm{I}-2.474)$ & & - & \\
\hline Others & $2.937(0.354-24.393)$ & & - & \\
\hline \multicolumn{5}{|l|}{ Tumor differentiation } \\
\hline Well differentiated & I (reference) & & I (reference) & \\
\hline Moderately or poorly differentiated & $7.453(3.445-16.125)$ & $<0.001$ & $7.453(1.911-17.662)$ & 0.002 \\
\hline \multicolumn{5}{|l|}{ Pre-CRT CEA (ng/mL) } \\
\hline$\leq 5$ & I (reference) & & - & \\
\hline$>5$ & $3.113(1.825-5.309)$ & $<0.001$ & - & \\
\hline \multicolumn{5}{|l|}{ Pre-CRT CAI 99 (U/mL) } \\
\hline$\leq 35$ & I (reference) & & - & \\
\hline$>35$ & $\mathrm{I} .487(0.727-3.043)$ & 0.277 & - & \\
\hline \multicolumn{5}{|l|}{ LMR } \\
\hline$\leq 4.91$ & I (reference) & & - & \\
\hline$>4.91$ & $0.79 \mid(0.47|-| .328)$ & 0.375 & - & \\
\hline
\end{tabular}

Notes: $P$-value; in logistic regression analysis, variables found to be statistically significant $(P<0.05)$ in univariate analysis were entered into a logistic regression multivariate model using a forward conditional method. '-' indicates multivariate analysis not performed.

Abbreviations: OR, odds ratio; $\mathrm{Cl}$, confidence interval; XELOX, oxaliplatin $130 \mathrm{mg} / \mathrm{m}^{2}$ administered intravenously on Day I and capecitabine administered orally I,000 $\mathrm{mg} / \mathrm{m}^{2}$ twice daily on Days I-I4 for a 3-week cycle; IMRT, intensity-modulated radiation therapy; 3D-CRT, 3-dimensional conformal radiation therapy; RT, radiotherapy; DAV, distance of inferior tumor margin from the anal verge; TNM, tumor node metastasis; CRT, chemoradiotherapy; CEA, carcinoembryonic antigen; CAI99, cancer antigen I99; LMR, lymphocyte-to-monocyte ratio.

et al reported that inflammatory monocytes may contribute to cancer development. ${ }^{22}$ Ozawa et al also reported that macrophages from within the tumor or those in peripheral locations and in the precursor blood of tumor-bearing mice are all targets of immunosuppressive strategies in tumor hosts, and within developing tumors, macrophages can help the progression of the disease. ${ }^{11}$ Finally, a growing amount of evidence indicates that tumor growth is closely associated 
A

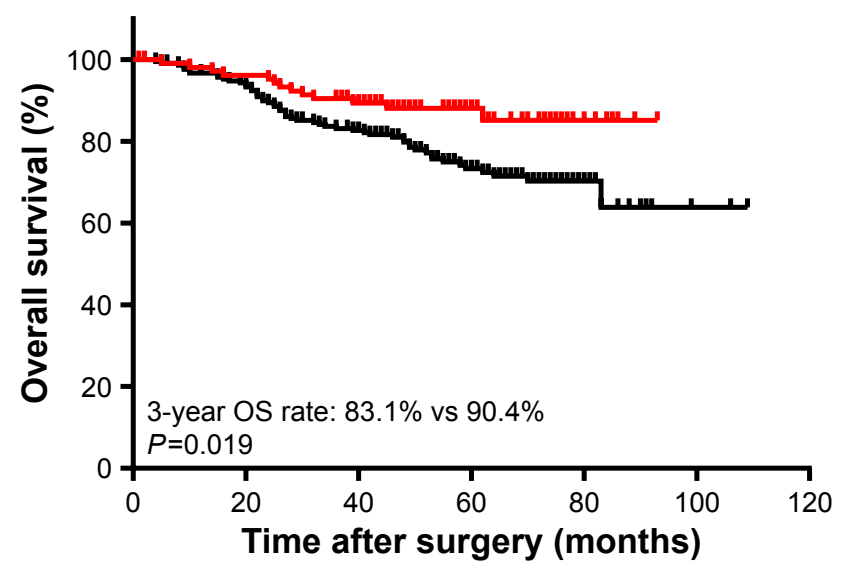

B

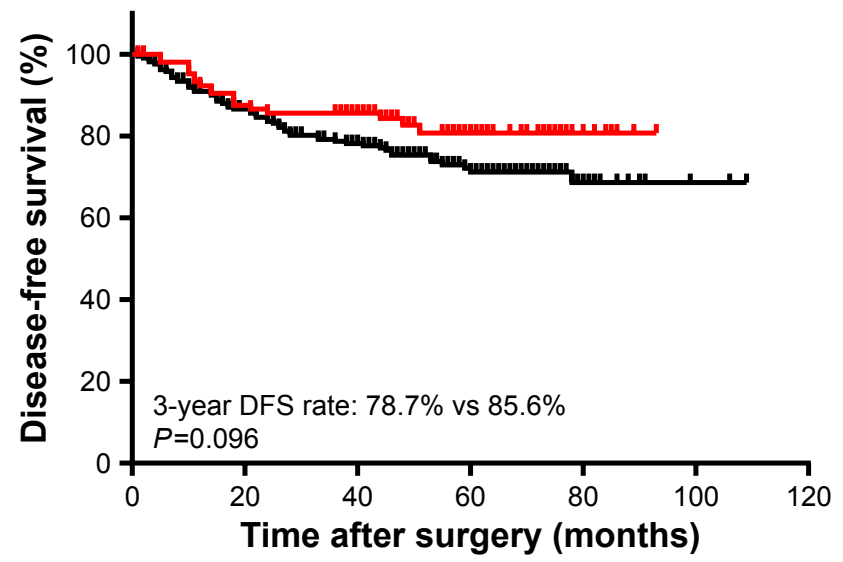

$$
\text { + High LMR }(n=107) \text { Low LMR }(n=210)
$$

Figure 2 (A) Kaplan-Meier curves of LMR for 3-year OS, (B) Kaplan-Meier curves of LMR for 3-year DFS.

Abbreviations: LMR, lymphocyte-to-monocyte ratio; OS, overall survival; DFS, disease-free survival.

Table 3 The univariate and multivariate analyses of the factors influencing OS and DFS by the Cox proportional hazard model

\begin{tabular}{|c|c|c|c|c|c|c|c|c|}
\hline & \multicolumn{4}{|l|}{ OS } & \multicolumn{4}{|l|}{ DFS } \\
\hline & \multicolumn{2}{|l|}{ Univariate analysis } & \multicolumn{2}{|c|}{ Multivariate analysis } & \multicolumn{2}{|l|}{ Univariate analysis } & \multicolumn{2}{|c|}{ Multivariate analysis } \\
\hline & HR (95\% Cl) & $P$-value & HR (95\% Cl) & $P$-value & HR (95\% Cl) & $P$-value & HR (95\% Cl) & $P$-value \\
\hline \multicolumn{9}{|l|}{ Age (years) } \\
\hline$\leq 60$ & I (reference) & & - & & I (reference) & & - & \\
\hline$>60$ & $1.167(0.711-1.915)$ & $0.54 I$ & - & & $0.868(0.530-1.420)$ & 0.572 & - & \\
\hline \multicolumn{9}{|l|}{ Sex } \\
\hline Male & I (reference) & & - & & I (reference) & & - & \\
\hline Female & $0.874(0.525-1.457)$ & 0.606 & - & & 1.095 (0.685-I.752) & 0.704 & - & \\
\hline \multicolumn{9}{|c|}{ Cycles of preoperative XELOX regimen } \\
\hline $\mathrm{I}-2$ & I (reference) & & - & & I (reference) & & - & \\
\hline $3-4$ & $0.93 \mid(0.566-1.559)$ & 0.785 & - & & $1.007(0.628-1.615)$ & 0.977 & - & \\
\hline \multicolumn{9}{|c|}{ Radiotherapy model } \\
\hline IMRT & I (reference) & 0.500 & - & & I (reference) & 0.732 & - & \\
\hline 3D-CRT & I.I0I (0.646-I.876) & & - & & $1.005(0.618-1.634)$ & & - & \\
\hline Conventional RT & $1.685(0.705-4.026)$ & & - & & $1.406(0.582-3.395)$ & & - & \\
\hline \multicolumn{9}{|c|}{ Radiotherapy dose (Gy) } \\
\hline 46 & I (reference) & & - & & I (reference) & & - & \\
\hline 50 & $0.922(0.806-1.054)$ & 0.231 & - & & $0.787(0.486-1.276)$ & 0.331 & - & \\
\hline \multicolumn{9}{|c|}{ Interval from radiotherapy to surgery (days) } \\
\hline$\leq 49$ & I (reference) & & - & & I (reference) & & - & \\
\hline$>49$ & $0.714(0.432-1.180)$ & 0.189 & - & & $0.658(0.409-1.058)$ & 0.084 & - & \\
\hline \multicolumn{9}{|l|}{ Tumor size $(\mathrm{cm})$} \\
\hline$\leq 3$ & I (reference) & & - & & I (reference) & & I (reference) & \\
\hline$>3$ & $1.852(1.138-3.013)$ & 0.013 & - & & $2.035(1.284-3.225)$ & 0.002 & $1.927(1.214-3.059)$ & 0.005 \\
\hline \multicolumn{9}{|l|}{$\mathrm{DAV}(\mathrm{cm})$} \\
\hline$\leq 5$ & I (reference) & & - & & I (reference) & & - & \\
\hline$>5$ & $0.705(0.430-1.153)$ & 0.163 & - & & $1.067(0.676-1.683)$ & 0.782 & - & \\
\hline \multicolumn{9}{|c|}{ Postoperative TNM stage } \\
\hline PCR I-II & I (reference) & & I (reference) & & I (reference) & & I (reference) & \\
\hline III & $3.253(2.000-5.291)$ & $<0.001$ & $3.004(1.837-4.912)$ & $<0.001$ & $3.300(2.07 I-5.257)$ & $<0.001$ & $3.182(1.996-5.075)$ & $<0.001$ \\
\hline \multicolumn{9}{|l|}{ TRG } \\
\hline $1-2$ & I (reference) & & - & & I (reference) & & - & \\
\hline $3-5$ & $2.062(1.245-3.414)$ & 0.005 & - & & $1.926(1.199-3.092)$ & 0.007 & - & \\
\hline
\end{tabular}


Table 3 (Continued)

\begin{tabular}{|c|c|c|c|c|c|c|c|c|}
\hline & \multicolumn{4}{|l|}{ OS } & \multicolumn{4}{|l|}{ DFS } \\
\hline & \multicolumn{2}{|l|}{ Univariate analysis } & \multicolumn{2}{|c|}{ Multivariate analysis } & \multicolumn{2}{|l|}{ Univariate analysis } & \multicolumn{2}{|c|}{ Multivariate analysis } \\
\hline & HR (95\% Cl) & $P$-value & HR (95\% Cl) & $P$-value & HR (95\% Cl) & $P$-value & HR (95\% Cl) & $P$-value \\
\hline \multicolumn{9}{|l|}{ Surgical type } \\
\hline Anterior resection & I (reference) & 0.017 & - & & I (reference) & 0.099 & - & \\
\hline $\begin{array}{l}\text { Abdominal perineal } \\
\text { resection }\end{array}$ & $1.702(1.037-2.795)$ & & - & & $1.347(0.837-2.168)$ & & - & \\
\hline Others & 3.503 (1.240-9.897) & & - & & 2.805 (1.004-7.834) & & - & \\
\hline \multicolumn{9}{|l|}{ Tumor differentiation } \\
\hline Well differentiated & I (reference) & & - & & I (reference) & & - & \\
\hline $\begin{array}{l}\text { Moderately or } \\
\text { poorly differentiated }\end{array}$ & $2.754(0.865-8.77 I)$ & 0.087 & - & & $2.393(0.873-6.555)$ & 0.090 & - & \\
\hline \multicolumn{9}{|l|}{ Pre-CRT CEA (ng/mL) } \\
\hline$\leq 5$ & I (reference) & & - & & I (reference) & & - & \\
\hline$>5$ & 1.581 (0.973-2.570) & 0.064 & & & $1.530(0.965-2.424)$ & 0.070 & - & \\
\hline \multicolumn{9}{|c|}{ Pre-CRT CAI99 (U/mL) } \\
\hline$\leq 35$ & I (reference) & & I (reference) & & I (reference) & & - & \\
\hline$>35$ & $2.332(1.38 I-3.940)$ & 0.002 & $1.906(1.116-3.254)$ & 0.018 & $2.025(1.212-3.384)$ & 0.007 & - & \\
\hline \multicolumn{9}{|l|}{ LMR } \\
\hline$\leq 4.91$ & I (reference) & & I (reference) & & I (reference) & & - & \\
\hline$>4.91$ & $0.493(0.269-0.904)$ & 0.022 & $0.538(0.292-0.991)$ & 0.047 & $0.640(0.376-1.089)$ & 0.100 & - & \\
\hline
\end{tabular}

Notes: $P$-value; in Cox hazards regression analysis, variables found to be statistically significant $(P<0.05)$ in univariate analysis were entered into a Cox regression multivariate model using a forward conditional method. '-' indicates multivariate analysis not performed.

Abbreviations: OS, overall survival; DFS, disease-free survival; $\mathrm{HR}$, hazard ratio, $\mathrm{Cl}$, confidence interval; XELOX, oxaliplatin I30 mg/m² administered intravenously on Day I and capecitabine administered orally I,000 mg/m² twice daily on Days I-I4 for a 3-week cycle; IMRT, intensity-modulated radiation therapy; 3D-CRT, 3-dimensional conformal radiation therapy; RT, radiotherapy; DAV, distance of inferior tumor margin from the anal verge; TNM, tumor node metastasis; $\mathrm{PCR}$, pathologic complete response; TRG, tumor regression grade; CRT, chemoradiotherapy; CEA, carcinoembryonic antigen; CAI99, cancer antigen I99; LMR, lymphocyte-to-monocyte ratio.

with myeloid cell recruitment, including macrophages. Under the influence of multiple microenvironmental signals, macrophages polarize towards phenotypes with pro-tumoral activities. ${ }^{23}$ Through our analysis, we found that tumortriggered inflammation resulted in reduced LMR. At the same time, systemic inflammation promoted cancer progression, eventually resulting in an unfavorable prognosis in LARC patients.

Our findings demonstrate that the LMR before CRT is an independent predictor of OS for patients with LARC. Our findings are consistent with previous studies on the relationship between the LMR and the prognosis of many other cancers, such as small cell lung cancer, ovarian cancer, esophageal cancer and nonmetastatic clear cell renal cell carcinoma. ${ }^{24-27}$

However, multivariate analysis in our study indicates that the LMR failed to play a predictive role in DFS. Similarly, researchers found that according to univariate analysis the LMR was not associated with recurrence-free survival. ${ }^{28}$ This might be explained by the fact that the LMR is unable to predict postoperative recurrence of LARC after preoperative CRT. ${ }^{29}$

For patients with LARC, we can use MRI, abdominal CT, CEA and genetic testing to determine the patient's disease progression. However, these tests are both expensive and inconvenient. The LMR is a convenient and low-cost marker that is not only reproducible but also easily accessible. Therefore, the LMR biomarker may be helpful in differentiating high-risk from low-risk disease and in determining the form of adjuvant therapy to use in order to achieve maximum therapeutic effectiveness.

Several limitations of the present study should be addressed. First, this is a retrospective study with an uncontrolled methodology. Second, only 317 patients were enrolled in our study, which may have resulted in bias during analysis. Third, appropriate cutoff levels of LMR were calculated for OS using the ROC analysis. In fact, these cutoff variables were not consistent with those in previous studies. Thus, a reasonable cutoff value that could be used to prospectively predict the prognosis of LARC should be identified. Finally, several disease conditions, such as infection, ischemia and trauma, which may bias the blood-circulating cell counts, were not taken into consideration.

\section{Conclusion}

The findings of our study indicate that a lower LMR before preoperative CRT is an independent prognostic biomarker for OS in LARC patients. Therefore, based on the level of LMR, 
the surgeon can assess the LARC patient's OS and formulate individualized strategies for preoperative treatment.

\section{Acknowledgments}

We deeply appreciate the help from all colleagues from the Department of Colorectal Surgery at Sun Yat-sen University Cancer Center, who obtained the blood specimens for this study. The authors would like to thank the two pathologists, Shixun Lu and Peng Li, from the Department of Pathology at Sun Yat-sen University Cancer Center for the TRG evaluation. This work was supported by grants from Science and Technology Planning Project of Guangdong Province (No. 2013B021800146), Natural Science Foundation of Guangdong Province (No 2017A030310204), Sun Yat-sen University Clinical Research 5010 Program (grant no 2015024) and National Natural Science Foundation of China (grant no 81772595). The authenticity of the data in this article has been validated by uploading the key raw data onto the Research Data Deposit public platform (www.researchdata.org.cn), with the approval number as RDDA2017000378.

\section{Disclosure}

The authors report no conflicts of interest in this work.

\section{References}

1. Ferlay J, Soerjomataram I, Dikshit R, et al. Cancer incidence and mortality worldwide: sources, methods and major patterns in GLOBOCAN 2012. Int J Cancer. 2015;136(5):E359-E386.

2. Bailey CE, Hu CY, You YN, et al. Increasing disparities in the age-related incidences of colon and rectal cancers in the United States, 1975-2010. JAMA Surg. 2015;150(1):17-22.

3. Yang Y, Huang X, Sun J, et al. Prognostic value of perineural invasion in colorectal cancer: a meta-analysis. J Gastrointest Surg. 2015;19(6): $1113-1122$

4. Glimelius B, Tiret E, Cervantes A, Arnold D; ESMO Guidelines Working Group. Rectal cancer: ESMO Clinical Practice Guidelines for diagnosis, treatment and follow-up. Ann Oncol. 2013;24(Suppl 6): vi81-vi88.

5. Fleming FJ, Påhlman L, Monson JR. Neoadjuvant therapy in rectal cancer. Dis Colon Rectum. 2011;54(7):901-912.

6. Gao YH, Lin JZ, An X, et al. Neoadjuvant sandwich treatment with oxaliplatin and capecitabine administered prior to, concurrently with, and following radiation therapy in locally advanced rectal cancer: a prospective phase 2 trial. Int J Radiat Oncol Biol Phys. 2014;90(5): 1153-1160.

7. Bhangu A, Wood G, Brown G, Darzi A, Tekkis P, Goldin R. The role of epithelial mesenchymal transition and resistance to neoadjuvant therapy in locally advanced rectal cancer. Colorectal Dis. 2014;16(4): O133-O143.

8. Balkwill F, Mantovani A. Inflammation and cancer: back to Virchow? Lancet. 2001;357(9255):539-545.

9. Mantovani A, Allavena P, Sica A, Balkwill F. Cancer-related inflammation. Nature. 2008;454(7203):436-444.
10. Chan JC, Chan DL, Diakos CI, et al. The lymphocyte-to-monocyte ratio is a superior predictor of overall survival in comparison to established biomarkers of resectable colorectal cancer. Ann Surg. 2017; 265(3):539-546.

11. Ozawa $\mathrm{T}$, Ishihara $\mathrm{S}, \mathrm{Kawai} \mathrm{K}$, et al. Impact of a lymphocyte to monocyte ratio in stage IV colorectal cancer. J Surg Res. 2015;199(2):386-392.

12. Mandard AM, Dalibard F, Mandard JC, et al. Pathologic assessment of tumor regression after preoperative chemoradiotherapy of esophageal carcinoma. Clinicopathologic correlations. Cancer. 1994;73(11): 2680-2686.

13. Coussens LM, Werb Z. Inflammation and cancer. Nature. 2002; 420(6917):860-867.

14. Wu Y, Zhou BP. Inflammation: a driving force speeds cancer metastasis. Cell Cycle. 2009;8(20):3267-3273.

15. Martínez-Lostao L, Anel A, Pardo J. How do cytotoxic lymphocytes kill cancer cells? Clin Cancer Res. 2015;21(22):5047-5056.

16. Hoffmann TK, Dworacki G, Tsukihiro T, et al. Spontaneous apoptosis of circulating T lymphocytes in patients with head and neck cancer and its clinical importance. Clin Cancer Res. 2002;8(8):2553-2562.

17. Dunn GP, Old LJ, Schreiber RD. The immunobiology of cancer immunosurveillance and immunoediting. Immunity. 2004;21(2):137-148.

18. Rabinowich H, Cohen R, Bruderman I, Steiner Z, Klajman A. Functional analysis of mononuclear cells infiltrating into tumors: lysis of autologous human tumor cells by cultured infiltrating lymphocytes. Cancer Res. 1987;47(1):173-177.

19. Menges T, Engel J, Welters I, et al. Changes in blood lymphocyte populations after multiple trauma: association with posttraumatic complications. Crit Care Med. 1999;27(4):733-740.

20. Kitayama J, Yasuda K, Kawai K, Sunami E, Nagawa H. Circulating lymphocyte number has a positive association with tumor response in neoadjuvant chemoradiotherapy for advanced rectal cancer. Radiat Oncol. 2010;5:47.

21. Neofytou K, Smyth EC, Giakoustidis A, et al. The preoperative lymphocyte-to-monocyte ratio is prognostic of clinical outcomes for patients with liver-only colorectal metastases in the neoadjuvant setting. Ann Surg Oncol. 2015;22(13):4353-4362.

22. Augier S, Ciucci T, Luci C, Carle GF, Blin-Wakkach C, Wakkach A. Inflammatory blood monocytes contribute to tumor development and represent a privileged target to improve host immunosurveillance. J Immunol. 2010;185(12):7165-7173.

23. Galdiero MR, Bonavita E, Barajon I, Garlanda C, Mantovani A, Jaillon S. Tumor associated macrophages and neutrophils in cancer. Immunobiology. 2013;218(11):1402-1410.

24. Go SI, Kim RB, Song HN, et al. Prognostic significance of the lymphocyte-to-monocyte ratio in patients with small cell lung cancer. Med Oncol. 2014;31(12):323.

25. Eo WK, Chang HJ, Kwon SH, et al. The lymphocyte-monocyte ratio predicts patient survival and aggressiveness of ovarian cancer. $J$ Cancer. 2016;7(3):289-296.

26. Hirahara N, Matsubara T, Mizota Y, Ishibashi S, Tajima Y. Prognostic value of preoperative inflammatory response biomarkers in patients with esophageal cancer who undergo a curative thoracoscopic esophagectomy. BMC Surg. 2016;16(1):66.

27. Hutterer GC, Stoeckigt C, Stojakovic T, et al. Low preoperative lymphocyte-monocyte ratio (LMR) represents a potentially poor prognostic factor in nonmetastatic clear cell renal cell carcinoma. Urol Oncol. 2014;32(7):1041-1048.

28. Dorofeeva NA, Grigorieva YS, Nikitina LS, et al. Effects of ERK1/2 kinases inactivation on the nigrostriatal system of KrushinskyMolodkina rats genetically prone to audiogenic seizures. Neurol Res. 2017;39(10):918-925.

29. Wu QB, Wang M, Hu T, He WB, Wang ZQ. Prognostic role of the lymphocyte-to-monocyte ratio in patients undergoing resection for nonmetastatic rectal cancer. Medicine (Baltimore). 2016;95(44):e4945. 
OncoTargets and Therapy

\section{Publish your work in this journal}

OncoTargets and Therapy is an international, peer-reviewed, open access journal focusing on the pathological basis of all cancers, potential targets for therapy and treatment protocols employed to improve the management of cancer patients. The journal also focuses on the impact of management programs and new therapeutic agents and protocols on

patient perspectives such as quality of life, adherence and satisfaction. The manuscript management system is completely online and includes a very quick and fair peer-review system, which is all easy to use. Visit http://www.dovepress.com/testimonials.php to read real quotes from published authors.

Submit your manuscript here: http://www.dovepress.com/oncotargets-and-therapy-journal 